\title{
Argila bentonítica funcionalizada com potássio: caracterização e uso como catalisar para reação de transesterificação do óleo de algodão
}

\author{
Jonei Marques da Costa ${ }^{1}$ (1) \\ Luiz Rogério Pinho de Andrade Lima ${ }^{1 *}$ (D)
}

\section{Resumo}

O uso de argilas é uma alternativa tecnicamente adequada à síntese de catalisadores de alta eficiência para transesterificação com o objetivo de produzir biodiesel etílico ou metílico, com vantagens em relação aos métodos comercialmente utilizados. O presente trabalho teve como objetivo analisar diferentes formas de funcionalizar uma bentonita com $\mathrm{K}^{+}$para catalisar a transesterificação etílica e metílica do óleo de algodão. A bentonita e catalisadores foram caracterizados por DRX, FRX e FT-IR. O óleo e seus produtos foram analisados por cromatografia gasosa e FT-IR. A análise exploratória da fração oleosa por FT-IR produziu resultados consistentes e convergentes com o método tradicional de análise por cromatografia gasosa. A transesterificação metílica teve seu melhor desempenho (91\%) usando o catalisador $\mathrm{KC} 400$ (Bentonita $+\mathrm{K}_{2} \mathrm{CO}_{3}$ ). A transesterificação etílica teve seu melhor desempenho (35\%) usando o catalisador KF700 (Bentonita + KF).

Palavras-chave: Argila; Caracterização; Materiais funcionalizados; Catalisador.

\section{Bentonitic clay functioned with potassium: characterization and use as a catalyst for cotton oil transesterification reaction}

\begin{abstract}
The use of clays is a technically suitable alternative to the synthesis of high-performance catalysts for transesterification in order to produce biodiesel ethyl or methyl, with advantages over the commercially used methods. This study aimed to analyze different ways to functionalize one bentonite $\mathrm{K}^{+}$to catalyze the transesterification ethyl and methyl cotton oil. Bentonite and catalysts were characterized by XRD, XRF and FT-IR. Oil and it is reaction products were analyzed by gas chromatography and FT-IR. Exploratory analysis of the oily fraction produced by FT-IR consistent results and converging with the traditional method of analysis by gas chromatography. Methyl transesterification performed best (91\%) using the $\mathrm{KC} 400$ catalyst (Bentonite $+\mathrm{K}_{2} \mathrm{CO}_{3}$ ). Ethyl transesterification had its best performance (35\%) using the catalyst KF700 (Bentonite $+\mathrm{KF})$.
\end{abstract}

Keywords: Clay; Characterization; Functionalized materials; Catalyst.

\section{Introdução}

As argilas in natura ou funcionalizadas podem ser utilizadas como catalisadores heterogêneos em diversas reações orgânicas. Em especial, seu uso na reação de transesterificação de óleos e gorduras para produção de biodiesel apresenta algumas vantagens como o baixo custo das argilas, simplicidade nos métodos de síntese do catalisador, possibilidade de reciclagem das argilas e redução de etapas de purificação do biodiesel [1,2].

Alguns estudos vêm utilizando fontes de $\mathrm{K}^{+}$como $\mathrm{KOH}, \mathrm{KF}, \mathrm{K}_{2} \mathrm{CO}_{3}, \mathrm{KNO}_{3}$ e $\mathrm{KI}$ para produzir catalisadores com argilas para transesterificação [3]. A transesterificação metílica de óleo vegetais catalisada por argilominerais funcionalizados com $\mathrm{KOH}$ [4-6], $\mathrm{K}_{2} \mathrm{CO}_{3}[7,8]$ e $\mathrm{KF}[9,10]$, vem obtendo bons resultados de conversão com reação a temperaturas brandas. Entretanto, não são observados muitos estudos comparativos entre as variáveis dos métodos de síntese dos catalisadores com argila e seus efeitos no desempenho da transesterificação.

As conversões são notadamente baixas na transesterificação etílica em temperatura inferior a $100^{\circ} \mathrm{C}$, como as observadas com uso de $\mathrm{KF} / \mathrm{Al}_{2} \mathrm{O}_{3}$ a $60^{\circ} \mathrm{C}$ com óleo de canola [3] e com uso de $\mathrm{KF} /$ Bentonita a $100{ }^{\circ} \mathrm{C}$ [11].

${ }^{\prime}$ Departamento de Ciência e Tecnologia dos Materiais, Universidade Federal da Bahia-UFBA, Salvador, BA, Brasil.

*Autor correspondente: lelo@ufba.br

2176-1523 C 2021. Costa et al. Publicado pela ABM. Este é um artigo publicado em acesso aberto (Open Access) sob a licença Creative Commons Attribution, que permite uso, distribuição e reprodução em qualquer meio, sem restrições desde que o trabalho original seja corretamente citado. 
Maiores conversão (90\%) foram obtidas com KF/Bentonita a temperatura reacional de $300^{\circ} \mathrm{C}$, em reator continuo.

A transesterificação do óleo de caroço de algodão é muito atrativo, visto que que este é um subproduto da cotonicultura [12]. No entanto, as investigações sobre o uso de argilas como catalisador para a transesterificação etílica ou metílica do óleo de semente de algodão precisam ser aprofundadas [13].

Poucas investigações são dedicadas a comparar o desempenho da transesterificação etílica e metílica catalisada por bentonitas funcionalizadas por diversas fontes de $\mathrm{K}+$, sobretudo, para a transesterificação do óleo de algodão catalisada por argilas funcionalizadas. Desta forma, este trabalho pretende contribuir apresentando um estudo mais aprofundado sobre a transesterificação etílica e metílica em diferentes condições reacionais do óleo de algodão catalisada por argila funcionalizada com $\mathrm{KF}, \mathrm{K}_{2} \mathrm{CO}_{3} \mathrm{e} \mathrm{KOH}$.

\section{Materiais e métodos}

Neste trabalho foi utilizada uma argila bentonita da Companhia Brasileira de Bentonita Ltda (CBB). Esta argila foi pulverizada $(<147 \mu \mathrm{m})$ e seca a $60{ }^{\circ} \mathrm{C}$ por $24 \mathrm{~h}$. Para funcionalização da bentonita foram utilizados o KF (Synth: 99\%), $\mathrm{K}_{2} \mathrm{CO}_{3}$ (Synth: 99\%), KOH (Synth: 96\%) e água deionizada $(<2 \mu \mathrm{S} / \mathrm{cm})$. Nos ensaios de transesterificação foi utilizado óleo de algodão comercial da ICOFORT Agroindustrial LTDA, álcool etílico (Êxodo: 95\%) e álcool metílico (Êxodo: 98\%).
Na síntese do catalisador foram utilizadas soluções de $\mathrm{KF}, \mathrm{K}_{2} \mathrm{CO}_{3}$ e $\mathrm{KOH}$ à $2 \mathrm{~mol} / \mathrm{L}$, sendo aplicada em cada uma das soluções uma dispersão aquosa a 10\% em massa de argila, mantida em reator com refluxo e controle de temperatura a $90{ }^{\circ} \mathrm{C}$. Em seguida, a mistura foi desidratada a $100{ }^{\circ} \mathrm{C}$ por $12 \mathrm{~h}$ em rotoevaporador. A pasta formada foi seca a $60^{\circ} \mathrm{C} \mathrm{e}$ pulverizada $(<147 \mu \mathrm{m})$; sendo posteriormente aquecida a 400 e $700{ }^{\circ} \mathrm{C}$ por $3 \mathrm{~h}$, seguido de resfriamento. Os catalisadores foram denominados por $\mathrm{KF}, \mathrm{KC}$ e $\mathrm{KH}$ quando sintetizados com $\mathrm{KF}, \mathrm{K}_{2} \mathrm{CO}_{3}$ e $\mathrm{KOH}$, respectivamente. O valor numérico inserido após o código representa a temperatura de síntese a $400{ }^{\circ} \mathrm{C}(\mathrm{KF} 400, \mathrm{KC} 400$ e $\mathrm{KH} 400)$ ou $700{ }^{\circ} \mathrm{C}(\mathrm{KF} 700$, $\mathrm{KC} 700$ e KH700).

A transesterificação foi realizada com catalisador na forma de pó $(<147 \mu \mathrm{m})$, em reator de vidro com agitação e refluxo a $70^{\circ} \mathrm{C}$ por $2 \mathrm{~h}$ (temperatura da reação estabilizada).

As condições reacionais estão sumarizadas nas Tabelas 1. A fase oleosa foi lavada com água deionizada, centrifugada e desidratada a $100{ }^{\circ} \mathrm{C}$ por $6 \mathrm{~h}$.

$\mathrm{Na}$ caracterização mineralógica dos catalisadores e bentonita foi utilizado um difratômetro da marca Shimadzu XRD-6100 (DRX) com catodo de cobre $\left(2^{\circ} / \mathrm{min}, 3^{\circ}-70^{\circ}\right.$, $40 \mathrm{kV}$ e $40 \mathrm{~mA}$ ). A identificação dos picos foi realizada com auxílio dos padrões cristalográficos disponíveis em Crystallography Open Database [14] e padrões cristalinos de compostos inorgânicos disponíveis em Materials Project [15].

Entre as funções disponíveis para representar o perfil de difração foi escolhida a função pseudo-Voigt modificada, na qual é possível a interpretação física do tipo de alargamento Gaussiano e Lorentziano. Os alargamentos Lorentzianos (wL)

Tabela 1. Parâmetros para transesterificação

\begin{tabular}{ccccc}
\hline Ensaios & Catalisador & Álcool & \% catalisador & $\begin{array}{c}\text { Razão molar } \\
\text { (álcool/óleo) }\end{array}$ \\
\hline 1 & Argila & Metanol & 15 & $10 / 1$ \\
2 & KF400 & Metanol & 20 & $20 / 1$ \\
3 & KF400 & Metanol & 10 & $05 / 1$ \\
4 & KC700 & Metanol & 20 & $10 / 1$ \\
5 & KC400 & Metanol & 20 & $10 / 1$ \\
6 & KC400 & Metanol & 10 & $10 / 1$ \\
7 & KH400 & Metanol & $10 / 1$ \\
8 & KF400 & Etanol & 10 & $10 / 1$ \\
9 & KF400 & Etanol & 5 & $20 / 1$ \\
10 & KF400 & Etanol & 10 & $10 / 1$ \\
11 & KF400 & Etanol & 20 & $10 / 1$ \\
12 & KF700 & Etanol & 10 & $10 / 1$ \\
13 & KF700 & Etanol & 20 & $10 / 1$ \\
14 & KH400 & Etanol & 5 & $10 / 1$ \\
15 & KH400 & Etanol & 10 & $20 / 1$ \\
16 & KH400 & Etanol & 10 & $05 / 1$ \\
17 & KH400 & Etanol & 20 & $10 / 1$ \\
18 & KH700 & Etanol & 5 & $10 / 1$ \\
21 & KH700 & Etanol & 10 & $20 / 1$ \\
20 & KH700 & Etanol & 20 & $10 / 1$ \\
& Etanol & 10 & $10 / 1$ \\
\end{tabular}


fornecem informações sobre os tamanhos dos cristalitos e os alargamentos Gaussianos (wG) fornecem informações sobre as deformações do cristalito [16].

$\mathrm{Na}$ identificação das ligações químicas nos catalisadores foi utilizado um espectrômetro de infravermelho da marca Jasco (FT-IR), modelo com uso de pastilha de $\mathrm{KBr}$ e resolução de $2 \mathrm{~cm}^{-1}$. A fluorescência de raios-X (FRX) utilizou um Bruker S2 Ranger com pastilha prensada em ácido esteárico.

O uso dos indicadores de Hammett permite determinar a força básica de um catalisador sólido. Apesar da limitação deste método é possível obter resultados comparativos de grande utilidade prática $[17,18]$. Foi agitado $0,01 \mathrm{~g}$ da amostra em $3 \mathrm{~mL}$ de etanol, com adição de $1 \mathrm{~mL}$ da solução dos indicadores Hammett $(0,1 \mathrm{mg} / \mathrm{L}$ em etanol) e, após a agitação e repouso por $4 \mathrm{~h}$, foi anotada a cor. Os indicadores usados foram (H_): i) 4-cloroanilina ( $\left.\mathrm{pK}_{\mathrm{a}}=26,5\right)$; ii) 4-nitroanilina $\left(\mathrm{pK}_{\mathrm{a}}=18,4\right)$; iii) Tropaeolin- $\mathrm{O}\left(\mathrm{pK}_{\mathrm{a}}=11,0\right)$; iv) Fenolftaleína $\left.\left(\mathrm{pK}_{\mathrm{a}}=7,2\right) ; \mathrm{v}\right)$ Azul de bromotimol $\left(\mathrm{pK}_{\mathrm{a}}=7,2\right)$; vi) Vermelho neutro $\left(\mathrm{pK}_{\mathrm{a}}=6,8\right)$ e vii) Dimetil amarelo $\left(\mathrm{pK}_{\mathrm{a}}=3,3\right)$.

A salubridade de $\mathrm{K}^{+}$contidos no catalisador em etanol foi estimada. Uma dispersão a $5 \%$ de catalisador em etanol foi mantida em agitação e refluxo por $4 \mathrm{~h} \mathrm{a} 80{ }^{\circ} \mathrm{C}$.

Após o tempo de reação a dispersão foi filtrada e o sólido foi seco por $5 \mathrm{~h}$ a $100{ }^{\circ} \mathrm{C}$. O catalisador foi então caracterizado por FRX e a medida de basicidade por indicadores de Hammett.

A caracterização da transesterificação etílica foi realizada por espectros de reflexão total atenuada no infravermelho com transformada de Fourier em um equipamento da Jasco utilizando um detector MCTI (4000 a $700 \mathrm{~cm}^{-1}$ ) e resolução de $1 \mathrm{~cm}^{-1}$. Esta analise teve por objetivo identificar o deslocamento da banda vibracional do estiramento do grupo carbonila em torno de $1730 \mathrm{~cm}^{-1}$. A correlação entre este deslocamento com a quantificação de éster etílico não foi utilizado neste trabalho [19]. Para consolidar os resultados obtidos com os espectros de infravermelho foram realizadas algumas análises por cromatografia gasosa usando um cromatógrafo da Shimadzu, seguindo as orientações da ASTM D6584 e utilizando uma coluna capilar MET-Biodiesel com $15 \mathrm{~m}$ e padrões fornecidos pela Sigma-Aldrich.

A taxa de conversão foi estimada utilizando a Equação 1.

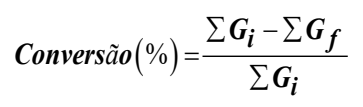

onde: $\mathrm{G}_{\mathrm{i}}$ é a soma dos glicerídeos (mono, di e tri) do óleo de algodão; $\mathrm{G}_{\mathrm{f}}$ é a soma dos glicerídeos (mono, di e tri) do produto das reações.

\section{Resultados e discussão}

\subsection{Caracterização química e mineralógica da argila bentonita}

A composição química da argila obtida for FRX estão apresentados na Tabela 2 e sugerem que a composição química da amostra é similar à montemorilonita, onde a presença de sílica, alumínio, ferro, magnésio e titânio fazem parte da estrutura cristalina e os demais elementos são cátions trocáveis.

O DRX da bentonita in natura e aquecidas podem ser visualizadas na Figura 1. Observa-se a presença de Mg-montemorilonita (ID COD: 9010956) e caulinita (ID COD: 1011045). As etapas de aquecimento a $400{ }^{\circ} \mathrm{C}$ e a $700{ }^{\circ} \mathrm{C}$, sugerem a formação de fases intermediárias de desidratação e desidroxilação. A Figura 2 mostra os alargamentos Lorentzianos (wL) e Gaussianos (wG), para o plano de reflexão característico d (001) da montemorilonita e caulinita.

O pico de reflexão máxima da montemorilonita d (001) foi analisado. Houve alteração no deslocamento do pico em $5,2^{\circ}$ na amostra in natura para $9,1^{\circ}$ a $400{ }^{\circ} \mathrm{C}$ e a $9,2^{\circ}$ a $700^{\circ} \mathrm{C}$.

A redução na posição característica do pico da montemorilonita pode ser atribuída à redução entre as camadas octaédrica e tetraédrica, devido à perda de umidade e desidroxilação parcial. As distâncias entre as camadas, obtidas teoricamente com a Lei de Bragg, foram de 16,9 $\AA$, $11,2 \AA$ e $9,6 \AA$, para montemorilonita in natura, aquecida a $400{ }^{\circ} \mathrm{C}$ e $700{ }^{\circ} \mathrm{C}$, respectivamente.

A degeneração do cristal em função da temperatura pode ser presumida pela análise do alargamento dos picos para um plano de reflexão específico. $\mathrm{O}$ valor de $\mathrm{wL}$ da caulinita reduziu de $0,78^{\circ}$ para $0,75^{\circ}$ quando aquecida a $400^{\circ} \mathrm{C}$, sugerindo que houve pouca alteração no tamanho do cristalito. O valor de $w G$ variou de $0,12^{\circ}$ para $2,5^{\circ}$ a $400^{\circ} \mathrm{C}$, sugerindo um aumento na tensão devido a deformação do cristalito. Com aquecimento a $700^{\circ} \mathrm{C}$ não é mais identificado o pico característico da caulinita devido a desidroxilação. O tamanho do cristalito da montemorilonita sofre redução a $400{ }^{\circ} \mathrm{C}$ e posteriormente aumento devido a desidroxilação parcial das camadas, como pode ser sugerido pela variação no valor de wL. O valor de wG sofre aumento contínuo com a variação da temperatura, indicando que o cristalito sofre intensa deformação a $700{ }^{\circ} \mathrm{C}$.

Os espectros de infravermelho da montemorilonita in natura (Ar), aquecida a $400{ }^{\circ} \mathrm{C}$ (AR400) e $700{ }^{\circ} \mathrm{C}$ (AR700), são visualizadas na Figura 3 . As bandas características da montemorilonita são atribuídas ao alongamento e flexão de $\mathrm{Si}-\mathrm{O}$ e Si-O-Si e são observadas em 1635, 1032, 1008 e 910 cm$^{-1}$.

Tabela 2. Composição química da argila in natura

\begin{tabular}{|c|c|c|c|c|c|c|c|c|c|c|c|}
\hline Pseudo-óxidos & $\mathrm{SiO}_{2}$ & $\mathrm{Al}_{2} \mathrm{O}_{3}$ & $\mathrm{Fe}_{2} \mathrm{O}_{3}$ & $\mathrm{MgO}$ & $\mathrm{TiO}_{2}$ & $\mathrm{CaO}$ & $\mathrm{BaO}$ & $\mathrm{Cr}_{2} \mathrm{O}_{3}$ & $\mathrm{~K}_{2} \mathrm{O}$ & $\mathrm{NiO}$ & Soma \\
\hline Concentração (\%) & 62,6 & 20,5 & 10,2 & 4,8 & 0,4 & 0,4 & 0,2 & 0,1 & 0,1 & 0,1 & 99,5 \\
\hline
\end{tabular}



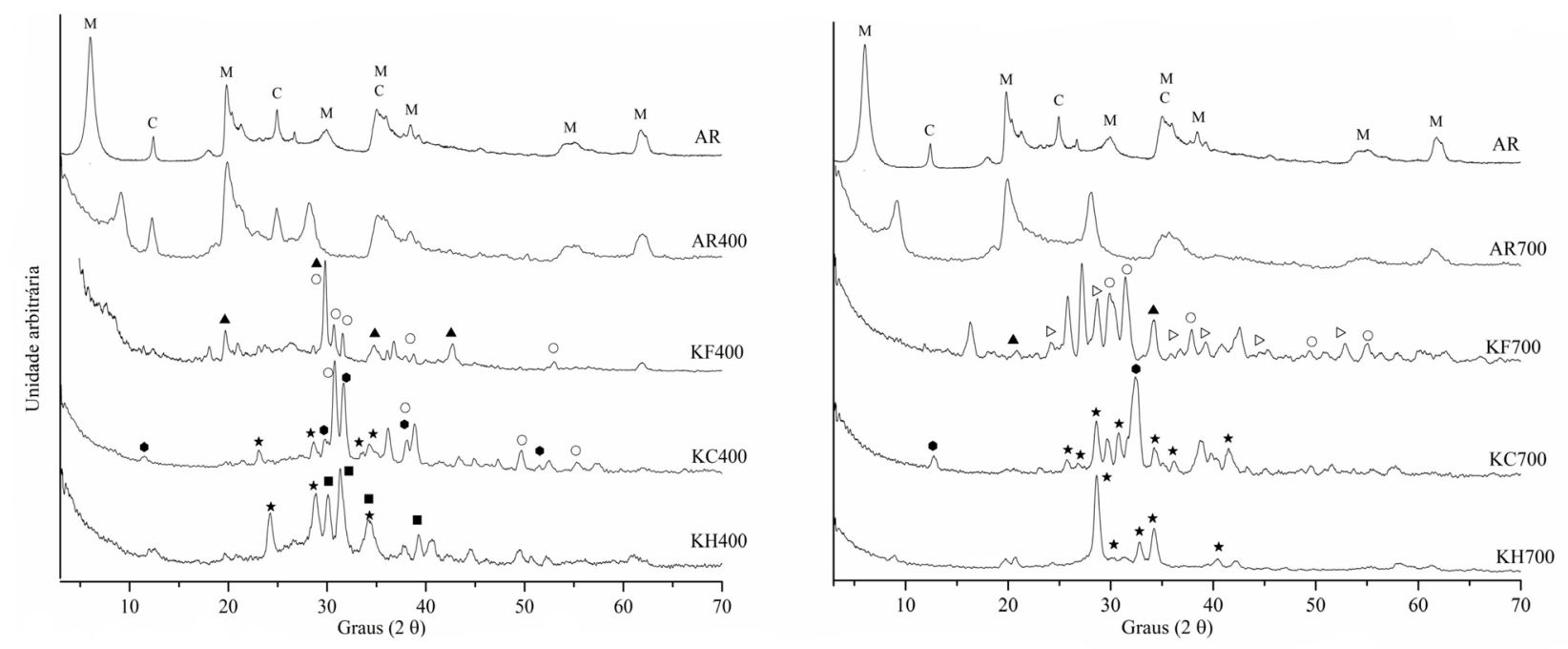

M: Montemorilonita. C: Caulinita. $\Delta \mathrm{KF} \cdot 6 \mathrm{H}_{2} \mathrm{O} \bullet \mathrm{K}_{2} \mathrm{CO}_{3} \boldsymbol{\nabla O H} \bigcirc \mathrm{K}_{2} \mathrm{O} \star \mathrm{KAlSiO}_{4} \triangleright \mathrm{KAlF}_{4}$

Figura 1. Padrões de difração da argila in natura (AR) e da argila funcionalizada a $400{ }^{\circ} \mathrm{C}(\mathrm{AR} 400, \mathrm{KF} 400, \mathrm{HC} 400$ e $\mathrm{AH} 400)$ e $700{ }^{\circ} \mathrm{C}(\mathrm{AR} 700$, KF700, HC700 e AH700).

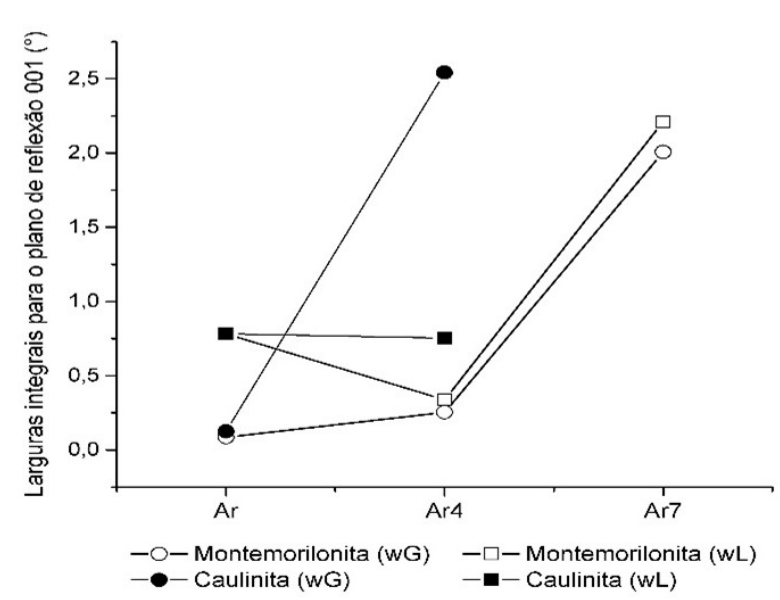

Figura 2. Valores da largura do pico característico d (001) da montemorilonita e caulinita.

As bandas vibracionais $3693 \mathrm{~cm}^{-1}$ representam o alongamento $\mathrm{O}-\mathrm{H}$ de grupos hidroxila da caulinita e a vibração $3620 \mathrm{~cm}^{-1}$ está relacionada ao alongamento O-H dos hidroxilos da rede da montemorilonita. Com o aquecimento a $400{ }^{\circ} \mathrm{C}$ não é percebido alterações nestas ligações. A $700^{\circ} \mathrm{C}$ a banda posicionada orginalmente a $3693 \mathrm{~cm}^{-1}$ se desloca para $3675 \mathrm{~cm}^{-1}$ e a banda posicionada a $3652 \mathrm{~cm}^{-1}$ torna-se mais intensa, sugerindo que houve ruptura das ligações $\mathrm{O}-\mathrm{H}$ interna da montemorilonita [20].

A banda em $1635 \mathrm{~cm}^{-1}$ atribuída a flexão de O-H da água intercalada, perde intensidade com o aquecimento [21]. As bandas em $915 \mathrm{~cm}^{-1}$ e $1116 \mathrm{~cm}^{-1}$ são degeneradas com o aquecimento até não serem mais visíveis a $700^{\circ} \mathrm{C}$. As bandas de absorção em 873, 832 e $693 \mathrm{~cm}^{-1}$ atribuídas às ligações metal-OH, se tornam indistinguíveis à medida que há aumento na temperatura, sugerindo que ocorreu à ruptura destas ligações [22]. Com o aquecimento a $700{ }^{\circ} \mathrm{C}$ o pico correspondente ao estiramento $\mathrm{Si}-\mathrm{O}-\mathrm{Si}$ em $1032 \mathrm{~cm}^{-1}$ sofre deformação atribuída à ruptura da ligação $\mathrm{Si}-\mathrm{OH}$ representada pela vibração em $1008 \mathrm{~cm}^{-1}$ que desaparece [22].

\subsection{Caracterização química e mineralógica das argilas funcionalizadas}

As fases cristalinas dos catalisadores sintetizados a 400 e a $700^{\circ} \mathrm{C}$ podem ser visualizadas na Figura 1.

Percebe-se que o uso de compostos com potássio promoveu alterações estruturais além das mudanças decorrente do aquecimento. Os catalisadores sintetizados a $400{ }^{\circ} \mathrm{C}$ apresentaram fases cristalinas da montemorilonita desidratada e alguns picos de reflexão da caulinita. Por sua vez, os catalisadores sintetizados a $700^{\circ} \mathrm{C}$ não exibem estes planos de reflexão. A formação de $\mathrm{KAlSiO}_{4}$ bem cristalizado deve ocorrer a temperatura de $950^{\circ} \mathrm{C}$ [23]. Entretanto, foram observados alguns planos de reflexão do $\mathrm{KAlSiO}_{4}$ quando a argila foi funcionalizada por carbonato de potássio e hidróxido de potássio e ativação a 400 e $700{ }^{\circ} \mathrm{C}$.

Os resultados sugerem que o fluoreto de potássio interage com as camadas da argila. É observado a possível presença de fluoreto de potássio não reagido e alguns planos de reflexão que sugerem a presença de $\mathrm{K}_{2} \mathrm{O}$, bem como, a presença de alguns picos de reflexão que não puderam ser plenamente identificados, presente no catalisador KF400 e KF700. No catalisador KF700 são observadas as fases cristalinas presentes no KF400 além da formação de $\mathrm{KAlF}_{4}$, decorrente da ruptura das ligações das camadas e interação com $\mathrm{F}^{-}$e $\mathrm{K}^{+}[9]$.

A presença de carbonato de potássio não reagido é observado no catalisador KC400 e KC700. No catalisador $\mathrm{KC} 400$, os planos de reflexão do carbonato de potássio são 


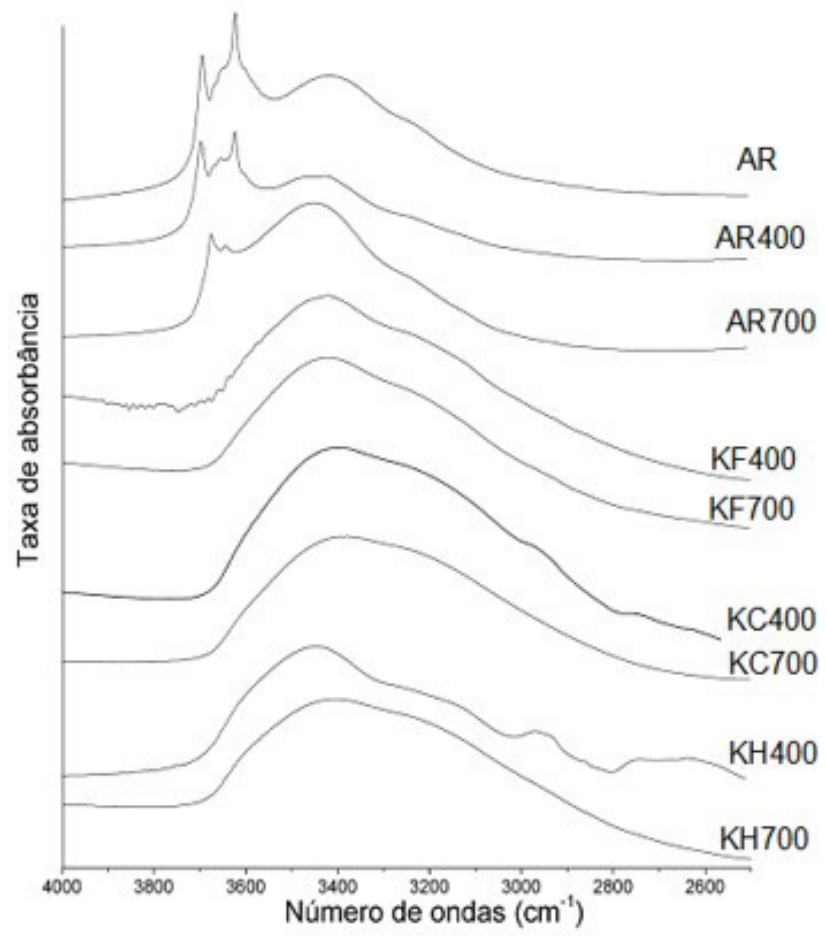

Figura 3. Espectros de infravermelho da argila e catalisadores.

bem definidos e a $700{ }^{\circ} \mathrm{C}$ estes picos são menos intensos, o que sugere maior consumo de reagente com aumento da temperatura. Os planos de reflexão característico do $\mathrm{KAlSiO}_{4}$ são mais nítidos com o aquecimento a $700{ }^{\circ} \mathrm{C}$, indicando que a desidroxilação permite maior interação do $\mathrm{K}^{+}$com aluminossilicato.

O hidróxido de potássio reage mais intensamente com a argila a $700{ }^{\circ} \mathrm{C}(\mathrm{KH} 700)$, formando a fase cristalina $\mathrm{KAlSiO}_{4}$ com planos de reflexão mais bem definidos. Podem ser observados planos de reflexão do $\mathrm{KOH} \mathrm{a} 400{ }^{\circ} \mathrm{C}$, devido à pouca interação com a argila.

Os espectros de FT-IR dos catalisadores são apresentados na Figura 3. As bandas de absorção entre 4000 a $3000 \mathrm{~cm}^{-1}$ são atribuídas as vibrações $\mathrm{O}-\mathrm{H}$ e as bandas entre 2000 e $1200 \mathrm{~cm}^{-1}$ dedicadas a compostos com carbonato [24].

As bandas atribuídas as ligações $\mathrm{O}-\mathrm{H}$ estrutural da montemorilonita e caulinita, atribuída a vibração 3693 e $3630 \mathrm{~cm}^{-1}$ são reduzidas com o processo de síntese dos catalisadores, sendo substituída por uma banda larga entre 3707 e $2600 \mathrm{~cm}^{-1}$, que pode ser atribuída a adsorção de água à superfície dos catalisadores [25]. A presença de carbonato de potássio não reagido no catalisador KC400 pode ser relacionada a pequenas vibrações em $1700 \mathrm{~cm}^{-1}$ [26].

A banda de maior intensidade em torno de $1000 \mathrm{~cm}^{-1}$ é atribuída a ligação Si-O. Com o aumento na temperatura de síntese do catalisador esta banda vibracional perde intensidade, que são mais intensas nos catalisadores KC700 e KH700. No catalisador KF400 e KF700 a banda de vibração em $430-450 \mathrm{~cm}^{-1}$ pode ser atribuída a presença da ligação K-F

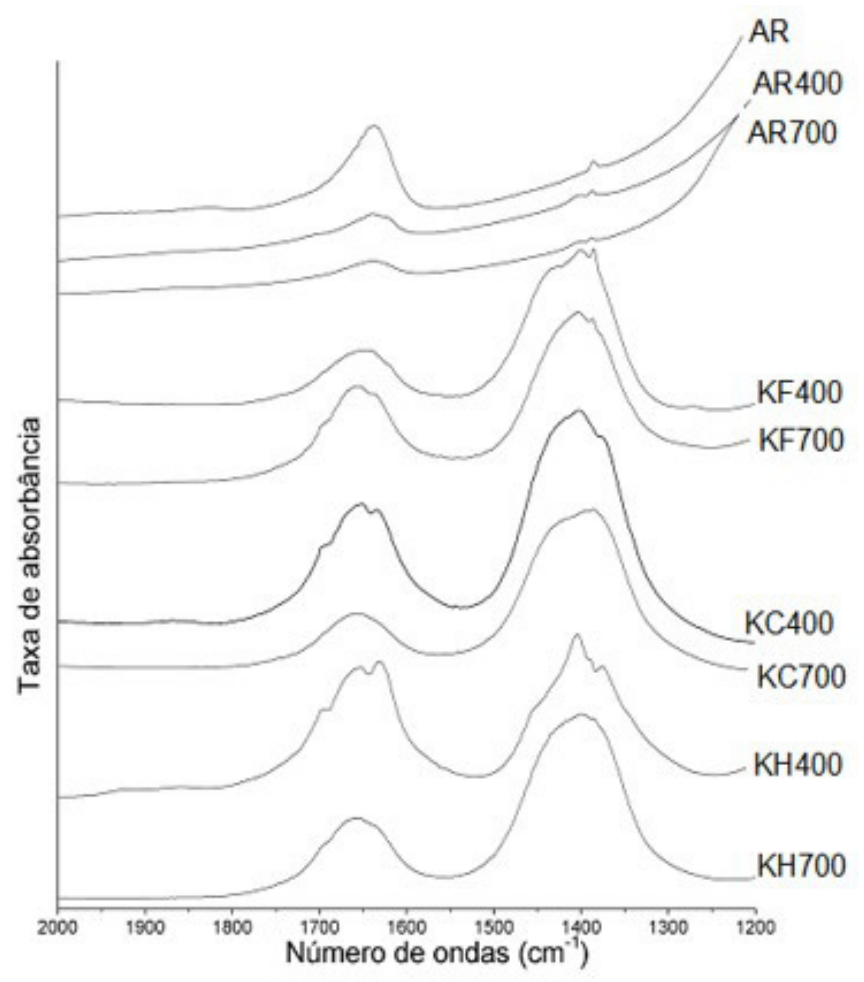

[27]. A interação Al-F, também relacionada a presença da fase cristalina $\mathrm{KAlF}_{4}$, é atribuída a vibração próximas a $607 \mathrm{~cm}^{-1}$ [27].

Os espectros de absorção do carbonato de potássio podem ser atribuídos às bandas de absorção próximas a 884 e $831 \mathrm{~cm}^{-1}$ e são observadas com maior intensidade no catalisador $\mathrm{KC} 400$ e de maneira menos intensa em KC700. A interação M-CO (M: Al, Fe e Mg) é indicada pela banda em $663 \mathrm{~cm}^{-1}$ [26]. A banda de absorção em $995 \mathrm{~cm}^{-1}$ é atribuída a ligação K-OH [28]. No catalisador KH400 esta banda é bem definida indicando que pouco reagente foi consumido, entretanto, no KH700 estas bandas são difusas sugerindo que todo o reagente foi consumido para formação de $\mathrm{KAlSiO}_{4}$ [29].

Percebe-se que o processo de tratamento com os íons $\mathrm{K}^{+}$produziram alterações na força básica da bentonita. A Argila in natura apresentou força básica ente $6,8<\mathrm{H}_{0}<3,3$, quando a bentonita foi aquecida a 700 e a $400{ }^{\circ} \mathrm{C}$ houve alteração na alcalinidade para $\mathrm{H}_{0}<3,3$. O KC40 tem a maior alcalinidade com $18,4<\mathrm{H}_{0}<26,5$ e o $\mathrm{KC} 700$ possui alcalinidade entre $9,8<\mathrm{H}_{0}<11,0$. O catalisador KF400 têm alcalinidade entre $15,0<\mathrm{H}_{0}<11,0$.

\subsection{Desempenho dos catalisadores}

A Figura 4 mostra os espectros em infravermelho na região da banda de vibração do alongamento do grupo carbonila para as diferentes condições de transesterificação etílica. A banda de estiramento $\mathrm{C}=\mathrm{O}$ do triglicerídeo, na 
amostra do óleo de algodão in natura, foi observada em $1743 \mathrm{~cm}^{-1}$. Com a formação de éster etílico esta banda deve ser deslocada gradualmente a $1735 \mathrm{~cm}^{-1}$.

O deslocamento máximo observado foi quando utilizado $10 \%$ do catalisador KC400 com razão molar de 10/1 (espectro 21), 10\% e 20\% do catalisador KF700 com razão molar 10/1 (espectro 12 e 13), sugerindo que estes catalisadores são mais eficientes. O catalisador $\mathrm{OH} 700$ nas mesmas condições (espectro 19) não apresentou deslocamento e o catalisador KC700 (espectro 22) sofreu um pequeno deslocamento para $1741 \mathrm{~cm}^{-1}$.

Os resultados da análise por cromatografia gasosa para estas reações, exibidas na Tabela 3, corrobora com as observações obtidas com os espectros de infravermelho, onde as reações realizadas com $10 \%$ de catalisador KF700 (espectro 12) e razão molar 10/1, KC700 (espectro 22) e KH700 (espectro 19) exibiram conversão de $35 \%$, $26 \%$ e $0 \%$; respectivamente.

Os resultados das análises do deslocamento da banda da carbonila sugerem que os melhores catalisadores são os sintetizados com fluoreto de potássio a $700{ }^{\circ} \mathrm{C}$ e carbonato de potássio a $400^{\circ} \mathrm{C}$. Não foi possível identificar a influência da razão molar no deslocamento da banda da carbonila em nenhum dos catalisadores. A concentração de catalisador contribuiu para melhorar o desempenho da reação, como observado na reação que usou 5\% de KF400 (espectro 8) que teve $2 \mathrm{~cm}^{-1}$ de deslocamento em comparação com a reação que usou $20 \%$ de KF400 (espectro 11) que o deslocamento foi de $5 \mathrm{~cm}^{-1}$.

A eficiência dos catalisadores na transesterificação etílica e metílica obtidas por cromatografia gasosa são apresentadas na Tabela 3. A transesterificação usando KF400 produziu baixa conversão com metanol e etanol. A conversão foi acentuada quando a razão molar metanol/óleo aumentou de 10/1 para 20/1 e a concentração de catalisador foi de $20 \%$. O catalisador KC400 produziu conversão superior a $90 \%$. O uso de $20 \%$ de catalisador com razão molar de 20/1 não proporcionou variação apreciável na conversão. Sendo assim, a razão molar de $5 / 1$ e $10 \%$ de catalisador é suficiente para a transesterificação com o KC400 [30], relata que é possível obter elevada conversão com zeólita impregnada com $\mathrm{K}_{2} \mathrm{CO}_{3}$ na transesterificação etílica [30].

A baixa conversão da transesterificação metílica observada com o uso dos catalisadores KC700 (22\%) e KH400 (32\%). A ausência de deslocamento da banda da carbonila observada nos espectros de infravermelho para o catalisador KH700 e KC700, sugerem que a formação dos cristalitos de $\mathrm{KAlSiO}_{4}$ não é favorável a transesterificação [31]. A baixa eficiência associada à presença de $\mathrm{KAlSiO}_{4}$, pode ser atribuída a peculiaridade da sua estrutura cristalina, visto
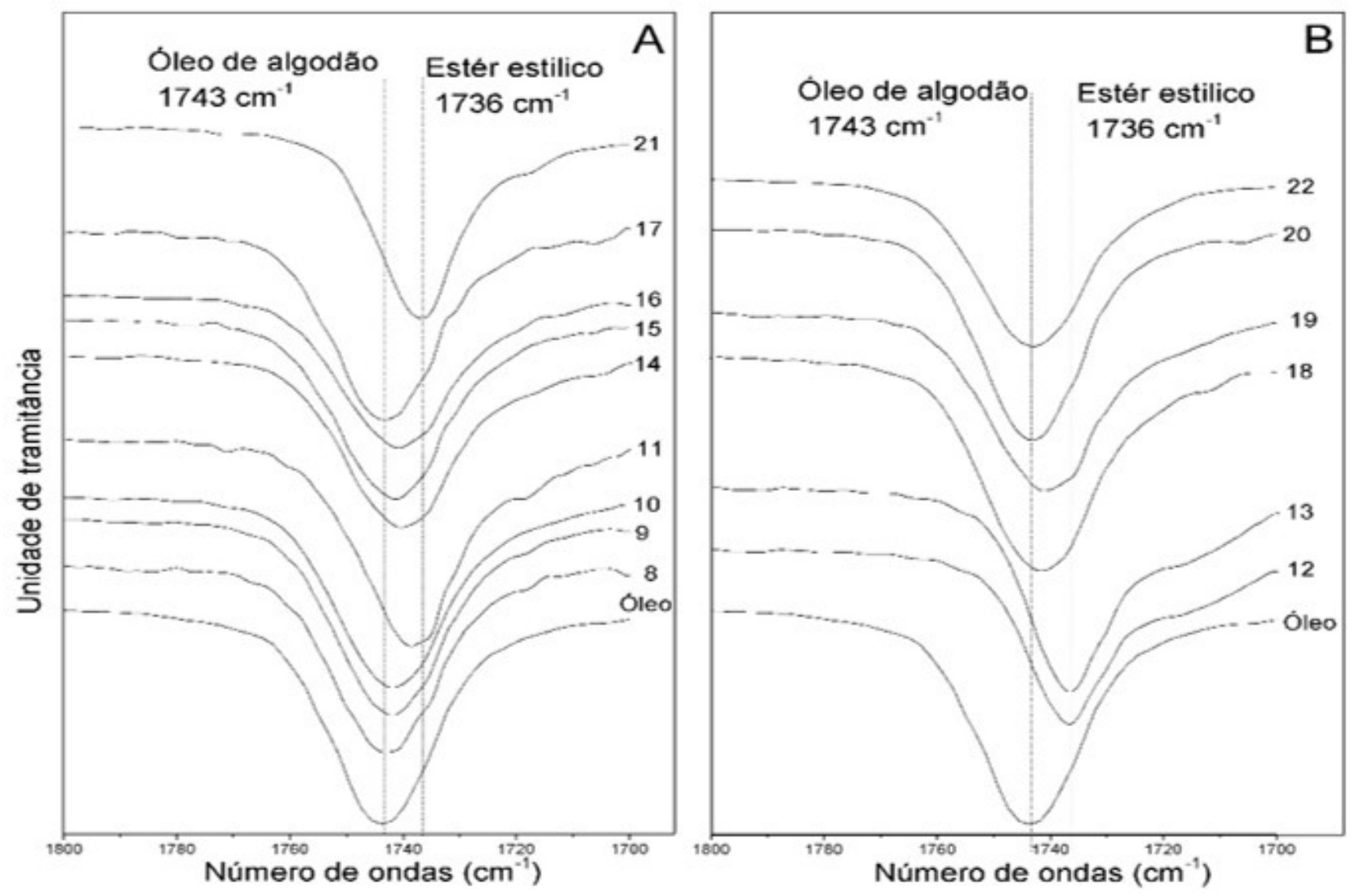

Figura 4. Espectros de infravermelho do óleo de algodão e dos óleos transesterificados com etanol. (A) catalisadores sintetizados a $400^{\circ} \mathrm{C}$; (B) catalisadores sintetizados a $700^{\circ} \mathrm{C}$. 
Tabela 3. Eficiência do catalisador

\begin{tabular}{cccccc}
\hline Ensaios & Álcool & Catalisador & \% catalisador & $\begin{array}{c}\text { Razão molar } \\
\text { (álcool/óleo) }\end{array}$ & Conversão (\%) \\
\hline 1 & Metanol & Argila & 15 & $10 / 1$ & 23 \\
2 & Metanol & KF400 & 20 & $20 / 1$ & 41 \\
3 & Metanol & KF400 & 10 & $05 / 1$ & 0 \\
4 & Metanol & KC700 & 20 & $10 / 1$ & 22 \\
5 & Metanol & KC400 & 20 & $10 / 1$ & 91 \\
6 & Metanol & KC400 & 10 & $05 / 1$ & 89 \\
7 & Metanol & KH400 & 10 & $10 / 1$ & 32 \\
12 & Etanol & KF700 & 10 & $10 / 1$ & 35 \\
19 & Etanol & KH700 & 10 & $10 / 1$ & 0 \\
22 & Etanol & KC700 & 10 & $10 / 1$ & 26 \\
\hline
\end{tabular}

que as bordas do cristal são constituídas de $\mathrm{AlO}_{4}$ e $\mathrm{SiO}_{4}$ que envolve o $\mathrm{K}^{+}[32]$.

A estabilidade química do catalisador KC400 foi investigada, visto apresentar melhor desempenho. Foi observado uma perda apreciável na concentração de $\mathrm{K}^{+}$. No catalisador KC400 a concentração de potássio era de $38,8 \%$. Após o processo de dissolução a concentração de potássio passou a $0,23 \%$. Entretanto, a análise de basicidade por indicadores de Hammett sugere que não houve alteração na força básica do catalisador, sendo mantida estável a 26,5 $<\mathrm{H}_{0}<18$,4. Alguns estudos relatam a aplicação de catalisadores sintetizados com até $45 \%$ de $\mathrm{K}_{2} \mathrm{CO}_{3}$ [30], entretanto não são observados relatos sobre a solubilidade dos íons de $\mathrm{K}^{+}$nos álcoois em condições análogas às reações $[30,33]$.

\section{Conclusões}

O uso de espectroscopia de infravermelho é uma alternativa consistente para uma análise exploratória da transesterificação etílica do óleo de algodão, permitindo uma resposta rápida e simples sobre a conversão de triglicerídeos a éster etílico.

Os processos de funcionalização da argila com íons de potássio, utilizando $\mathrm{K}_{2} \mathrm{CO}_{3}$ e $\mathrm{KOH}$, indica que a fase cristalina de $\mathrm{KAlSiO}_{4}$ é mais bem formada com aquecimento a $700{ }^{\circ} \mathrm{C}$ e sua presença sugere reduzir a eficiência da transesterificação. $\mathrm{O}$ uso de bentonita funcionalizada com $\mathrm{K}_{2} \mathrm{CO}_{3}$ a $400{ }^{\circ} \mathrm{C}$ proporcionou o melhor resultado para transesterificação metílica com $20 \%$ de catalisador e razão molar de 10/1. Os resultados sugerem que os íons $\mathrm{K}^{+}$presente no catalisador KC400 são solúveis em etanol em condições análogas à transesterificação, mas não promove alterações na força básica do catalisador.

$\mathrm{O}$ catalisador sintetizado com $\mathrm{KF}$ a $700{ }^{\circ} \mathrm{C}$ formou as

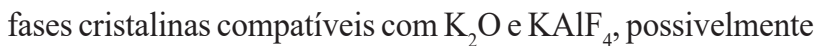
devido ao excesso de $\mathrm{F}^{-}$disponível, que pode ter promovido a sua reação com o $\mathrm{Al}^{+3}$ da rede cristalina da montemorilonita. A transesterificação etílica apresentou conversão máxima de $35 \%$ quando foi usado bentonita funcionalizada com KF, enquanto a metílica atingiu conversões de $90 \%$ quando foi usado bentonita funcionalizada com $\mathrm{K}_{2} \mathrm{CO}_{3}$.

\section{Agradecimentos}

Este trabalho foi realizado com apoio da Coordenação de Aperfeiçoamento de Pessoal de Nível Superior - Brasil (CAPES).

\section{Referências}

1 Sharma Y, Singh B, Korstad J. Latest developments on application of heterogenous basic catalysts for an efficient and eco friendly synthesis of biodiesel: a review. Fuel. 2011;90(4):1309-1324.

2 Hattori H. Solid base catalysts: Fundamentals and their applications in organic reactions. Applied Catalysis A, General. 2015;504:103-109.

3 Boz N, Kara M. Solid base catalyzed transesterification of canola oil. Chemical Engineering Communications. 2008;196(1-2):80-92.

4 Ballotin F, Nascimento M, Vieira S, Bertoli A, Carmignano O, Teixeira A, et al. Natural Mg silicates with different structures and morphologies: reaction with $\mathrm{K}$ to produce $\mathrm{K}_{2} \mathrm{MgSiO}_{4}$ catalyst for biodiesel production. International Journal of Minerals Metallurgy and Materials. 2020;27(1):46-54.

5 Agustian E. Savitri, Ghozali M, Wuryaningsih. Biodiesel production of jatropha curcas oil by bentonite as catalyst. Indonesian Institute of Sciences. In: Proceedings of the International Conference on Sustainable Energy Engineering and Application; 2012 Nov 6-8; Yogyakarta, Indonesia. Indonesia: Lembaga Ilmu Pengetahuan Indonesia; 2012. p. 35-39. 
6 Soetaredjo F, Ayucitra A, Ismadji S, Maukar A. KOH/bentonite catalysts for transesterification of palm oil to biodiesel. Applied Clay Science. 2011;53(2):341-346.

7 Boz N, Degirmenbasi N, Kalyon D. Transesterification of canola oil to biodiesel using calcium bentonite functionalized with K compounds. Applied Catalysis B: Environmental. 2013;138-139:236-242.

8 Degirmenbasi N, Boz N, Kalyon D. Biofuel production via transesterification using sepiolite-supported alkaline catalysts. Applied Catalysis B: Environmental. 2014;150-151:147-156.

9 Alves H, da Rocha A, Monteiro M, Moretti C, Cabrelon M, Schwengber C, et al. Treatment of clay with KF: new solid catalyst for biodiesel production. Applied Clay Science. 2014;91-92:98-104.

10 Silva L, Silva E, Monteiro M, Silva C, Teleken J, Alves H. Effect of the chemical composition of smectites used in KF/Clay catalysts on soybean oil transesterification into methyl esters. Applied Clay Science. 2014;102:121-127.

11 Santos K, Bariccatti R, De Rossi E, Colpini L, Eckert C, Dos Santos G, et al. Activity of heterogeneous catalysts in the methyl and ethyl transesterification of soybean oil. Journal of Food Agriculture and Environment. 2015;13(2):250-255.

12 Ribeiro M, Raiher A. Potentialities of energy generation from waste and feedstock produced by the agricultural sector in Brazil: the case of the State of Paraná. Energy Policy. 2013;60:208-216.

13 Sitepu E, Heimann K, Raston C, Zhang W. Critical evaluation of process parameters for direct biodiesel production from diverse feedstock. Renewable \& Sustainable Energy Reviews. 2020;123:109762.

14 Crystallography Open Database [página da internet]. 2020 [acesso em 2 mar. 2020]. Disponível em: http://www. crystallography.net/cod/

15 Persson K. Materials Project [Internet]. 2020 [acesso em 1 fev. 2020]. Disponível em: https://materialsproject.org/

16 Araújo J, Assis J, Monine V, Bertolino L. Caracterização da microestrutura de caulinitas pela difração de raios X. Matéria. 2006;11(3):361-371.

17 Yazici D, Bilgiç C. Determining the surface acidic properties of solid catalysts by amine titration using Hammett indicators and FTIR-pyridine adsorption methods. Surface and Interface Analysis. 2010;42(6-7):959-962.

18 Bernardo J. Catalisadores heterogéneos básicos para a produção de biodiesel [dissertação]. Lisboa: Instituto Superior de Engenharia de Lisboa; 2010.

19 Zagonel G, Peralta-Zamora P, Ramos L. Multivariate monitoring of soybean oil ethanolysis by FTIR. Talanta. 2004;63(4):1021-1025.

20 Caccamo M, Mavilia G, Mavilia L, Lombardo D, Magazù S. Self-assembly processes in hydrated montmorillonite by FTIR investigations. Materials. 2020;13(5):1100.

21 Tinti A, Tugnoli V, Bonora S, Francioso O. Recent applications of vibrational mid-Infrared (IR) spectroscopy for studying soil components: a review. Journal of Central European Agriculture. 2015;16(1):1-22.

22 Bishop J, Pieters C, Edwards J. Infrared spectroscopic analyses on the nature of water in montmorillonite. Clays and Clay Minerals. 1994;42(6):702-716.

23 Kawahara A, Andou Y, Marumo F, Okuno M. The crystal structure of high tempetature from of kalsilite $\left(\mathrm{KAlSiO}_{4}\right)$ at $950^{\circ} \mathrm{C}$. Mineralogical Journal. 1987;13:260-270.

24 Povnnennykh A. The use of infrared spectra for the determination of mineral. The American Mineralogist. 1978;63(2):956-959.

25 Madejová J. FT-IR techniques in clay mineral studies. Vibrational Spectroscopy. 2003;31(1):1-10.

26 Miller F, Wilkins C. Infrared spectra and characteristic frequencies of inorganic ions. Analytical Chemistry. 1952;28(6):1253-1294.

27 Liu Z, Wang J, Kang M, Yin N, Wang X, Tan Y, et al. Synthesis of glycerol carbonate by transesterification of glycerol and dimethyl carbonate over $\mathrm{KF} / \gamma-\mathrm{Al}_{2} \mathrm{O}_{3}$ catalyst. Journal of the Brazilian Chemical Society. 2013;25(1):152-160.

28 Ahmadpour A, Rashidi H, Mahboub M, Farmad M. Comparing the performance of KOH with NaOH-activated anthracites in terms of methane storage. Adsorption Science and Technology. 2013;31(8):729-745.

29 Parthasarathy G, Santosh M. Pressure induced polymorphic phase transition of natural metamorphic Kalsilite; electrical resistivity and infrared spectroscopic investigations. Minerals. 2015;5(4):647-653.

30 Silveira E, Perez V, Reyero I, Serrano-Lotina A, Justo O. Biodiesel production from heterogeneous catalysts based $\mathrm{K}_{2} \mathrm{CO}_{3}$ supported on extruded $\gamma-\mathrm{Al}_{2} \mathrm{O}_{3}$. Fuel. 2019;241:311-318. 
31 Wen G, Yan Z. Transesterification of soybean oil to biodiesel over Kalsilite catalyst. Frontiers of Chemical Science and Engineering. 2011;5(3):325-329.

32 Kremenović A, Lazic B, Krüger H, Tribus M, Vulić P. Monoclinic structure and nonstoichiometry of " $\mathrm{KAlSiO}_{4}-\mathrm{O} 1$ ". Acta Crystallographica. Section C, Crystal Structure Communications. 2013;69(4):334-336.

33 Shan R, Shi J, Yan B, Chen G, Yao J, Liu C. Transesterification of palm oil to fatty acids methyl ester using $\mathrm{K}_{2} \mathrm{CO}_{3} /$ palygorskite catalyst. Energy Conversion and Management. 2016;116:142-149.

Recebido em: 15 Ago. 2020

Aceito em: 9 Fev. 2021 